\title{
Analysis of gene expression profiling variations induced by hsa-miR-145-5p-overexpression in laryngeal squamous cell carcinoma cell line Tu-177
}

\author{
YONGXIA DING ${ }^{1,2}$, YONGYAN WU ${ }^{2,3}$, WEI GAO ${ }^{2,3}$, CHUNMING ZHANG $^{2,3}$, QINLI ZHAO $^{2,3}$, \\ HUINA GUO ${ }^{2,3}$, XUKUAN QU ${ }^{2,3}$, SHUXIN WEN $^{2,3}$ and BINQUAN WANG ${ }^{1-3}$ \\ ${ }^{1}$ Nursing College of Shanxi Medical University; ${ }^{2}$ Shanxi Key Laboratory of Otolaryngology, Head and Neck Cancer; \\ ${ }^{3}$ Department of Otolaryngology, Head and Neck Surgery, The First Hospital Affiliated with \\ Shanxi Medical University, Taiyuan, Shanxi 030001, P.R. China
}

Received April 26, 2017; Accepted July 12, 2017

DOI: $10.3892 / \mathrm{mmr} .2017 .7360$

\begin{abstract}
The present study aimed to investigate the variations of the gene network and biological functions induced by hsa-miR-145-5p in the laryngeal squamous cell carcinoma (LSCC) cell line Tu-177. A hsa-miR-145-5p-overexpressed Tu-177 cell model was established, and the gene expression microarray data of miR-145-5p-overexpressed cells and negative control (NC) cells were analyzed. The differentially expressed genes (DEGs) between two groups were identified, and their potential functions were predicted by functional enrichment analysis. Furthermore, the targets of miR-145-5p were identified from the DEGs, and their potential functions and protein-protein interactions (PPIs) were analyzed. The mRNA expressions of acetyl-CoA carboxylase $\beta(A C A C B)$, fibroblast growth factor receptor 1 (FGFR1), protein phosphatase 3 catalytic subunit a $(P P P 3 C A)$ and spleen associated tyrosine kinase $(S Y K)$, were analyzed via quantitative polymerase chain reaction. A total of 1,501 upregulated and 887 downregulated genes were identified in the hsa-miR-145-5p-overexpressed Tu-177 cells, compared with the NC cells. Of these DEGs, 164 upregulated and 221 downregulated genes were predicted to be targeted by
\end{abstract}

Correspondence to: Professor Binquan Wang, Department of Otolaryngology, Head and Neck Surgery, The First Hospital Affiliated with Shanxi Medical University, 85 Jiefang Road South, Taiyuan, Shanxi 030001, P.R. China

E-mail:wbq_xy@sxent.org

Abbreviations: LSCC, laryngeal squamous cell carcinoma; NC, negative control; DEGs, differentially expressed genes; PPIs, protein-protein interactions; GEO, Gene Expression Omnibus; RMA, Robust Multichip Average; GO, Gene Ontology; FC, fold-change; HNSCC, head and neck squamous cell carcinoma; CAMs, cell adhesion molecules; ACC, acetyl-CoA carboxylase; FGFR1, fibroblast growth factor receptor 1; BP, biological process

Key words: laryngeal squamous cell carcinoma, differentially expressed gene, microarray, pathway, network
hsa-miR-145-5p. The upregulated target genes were primarily associated with functions of immunity, whereas the downregulated target genes were significantly enriched in the p53 signaling pathway. In the PPI network consisting of 267 target genes, the upregulated $A C A C B$ had the greatest degree and interacted with downregulated genes including $P P P 3 C A$ and $S Y K$, in addition to upregulated genes, including FGFRl. The mRNA expressions of $A C A C B$ and FGFRlwere markedly enhanced in miR-145-5p-overexpressed Tu-177 cells, whereas overexpressing miR-145-5p significantly reduced mRNA expression of PPP3CA and SYK. hsa-miR-145-5p may exhibit an anticancer role in LSCC via regulating multiple cell processes, including cell proliferation and invasion, fatty acid metabolism, immunity and p53 signaling pathway. These findings provide novel information for the future investigation of miR-145-5p functions in LSCC.

\section{Introduction}

Laryngeal squamous cell carcinoma (LSCC) is one of the most common malignancies in head and neck (1). LSCC has been reported to occur more frequently in middle-aged and elderly men (1). In 2016, 13,430 new cases and 3,620 deaths are estimated of larynx cancer in United States (2). Currently, although marked developments have been achieved in terms of therapeutic strategies for LSCC, there are no such enhancements inthe therapeutic outcome and prognosis of patients with LSCC (2). Therefore, it is extremely urgent to uncover the molecular mechanisms of LSCC, which can contribute to improve the clinical therapy of LSCC.

Hsa-miR-145 is one of the most important tumor suppressor miRNAs, which is lowly expressed in various cancers, such as colorectal cancer (3), gallbladder cancer (4), pleural mesothelioma (5) andprostate cancer (6). In the researches on LSCC, miR-145 has also been shown to be downregulated, and overexpression of miR-145 inhibit the proliferation and metastasis in Hep-2 cells viainducing cell cycle arrest and apoptosis (7). A recent study has shown that miR-145 is able to inhibit stem cell potency of Hep-2 cells, along with the downregulation of stem cell marks like SOX2, KLF4, OCT4 and ABCG2 (8). 
Furthermore, we found that miR-145-5p was able to inhibit cell proliferation and metastasis in human LSCC cell line Tu-177, and downregulation of miR-145-5p led to poor prognosis of patients with LSCC (9). However, currently, the regulatory effect of miR-145-5p on the gene expression profiling in LSCC remains unclear.

In the present study, to reveal the variation of gene expression profiling induced by $\mathrm{miR}-145-5 \mathrm{p}$ overexpression in LSCC, miR-145-5p mimic was transfected into Tu-177 cells to generate miR-145-5p-overexpressed LSCC cells. Then, based on gene microarrays, differentially expressed genes (DEGs) between miR-145-5p-overexpressed Tu-177 cells and negative control (NC) cells were identified. The target genes of miR-145-5p among the DEGs were identified and their potential functions and protein-protein interactions (PPIs) were analyzed. These results were expected to be helpful for the better understanding of the effects of miR-145-5p on gene expressions in LSCC.

\section{Materials and methods}

Cell culture and establishment of hsa-miR-145-5p-overexpressed cell model. Human LSCC cell line Tu-177 was purchased from Shanghai Bioleaf Biotech Company (Shanghai, China). Cells were maintained in Roswell Park Memorial Institute-1640 (HyClone, Logan, UT, USA) containing $10 \%$ fetal bovine serum (Biological Industries, Cromwell, CT, USA) at $37^{\circ} \mathrm{C}$ in a humidified chamber supplemented with $5 \% \mathrm{CO}_{2}$. For miRNA mimic transfection, cells were plated in 6 -well dishes $\left(2.0 \times 10^{5}\right.$ cells per well). MiR-145-5p mimic and NC (GenePharma Co., Ltd, Shanghai, China) were transfected at a final concentration of $50 \mathrm{nM}$ by using Lipofectamine ${ }^{\circledR} 2000$ (Invitrogen, Carlsbad, CA, USA) according to the manufacturer's instructions. After $48 \mathrm{~h}$ of transfection, cells were harvested for the following procedures.

RNA extraction andarrayprocedures. TotalRNA was extracted from transfected cells using TRIzol reagent (Invitrogen) following the manufacturer's protocol. Double-strand cDNA (ds-cDNA) was synthesized from $5 \mu \mathrm{g}$ of total RNA using anSuperScript ds-cDNA synthesis kit (Invitrogen) in the presence of 100 pmol oligo dT primers. Subsequently, ds-cDNA was cleaned and labeled in accordance with the NimbleGen Gene Expression Analysis protocol (NimbleGen Systems, Inc., Madison, WI, USA). Briefly, ds-cDNA was incubated with $4 \mu \mathrm{g}$ RNase $\mathrm{A}$ at $37^{\circ} \mathrm{C}$ for $10 \mathrm{~min}$ and cleaned using phenol:chloroform:isoamyl alcohol, followed by ice-cold absolute ethanol precipitation. After purification, the generated cDNA was quantified using NanoDrop ND-1000 spectrophotometer (NanoDrop, Wilmington, DE, USA). Subsequently, the cDNA was labeled by a monochrome DNA labeling kit (NimbleGen Systems, Inc., Madison, WI, USA) following the manufacturer's protocol, and the labeled cDNA was hybridized withNimbleGen Human 12x135 k expression microarrays at $42^{\circ} \mathrm{C}$ for $16-20 \mathrm{~h}$. Finally, slides were washed using NimbleGen Wash Buffer kit (NimbleGen Systems, Inc.), and scanned by Axon GenePix 4000B microarray scanner (Axon Instruments Inc., Foster City, CA, USA). The microarray data were submitted to Gene Expression Omnibus (GEO) with an accession number GSE92678.
Real-time quantitative PCR ( $P P C R)$. First-strand cDNA was synthesized using All-in-One miRNA First-Strand cDNA Synthesis kit (GeneCopoeia, Inc., Rockville, MD, USA). The real-time quantitative PCR (RT-qPCR) was performed using ChamQ SYBR qPCR Master Mix (Vazyme, Piscataway, NJ, USA) on a ABI 7500 FAST real time PCR system (Applied Biosystems, Foster City, CA, USA). The procedures for the qPCR were as follows: $95^{\circ} \mathrm{C}$ for $30 \mathrm{sec}$, followed by 40 cycles of $95^{\circ} \mathrm{C}$ for $10 \mathrm{sec}$ and $60^{\circ} \mathrm{C}$ for $30 \mathrm{sec}$. The specificity of the primer amplicons was examined by the analysis of a melting curve. For miRNA-145-5p, the comparative Ct method was employed for quantification of target mRNA expression that was normalized to $\beta$-actin expression and relative to the calibrator. For $A C A C B, F G F R 1, P P P 3 C A$ and $S Y K$, the comparative $\mathrm{Ct}$ method was used for quantifying target mRNA expression normalized to that of $18 \mathrm{~S}$ rRNA. The forward primers used in qPCR are as follows: miR-145-5p-5'-GTCCAGTTTTCC CAGGAATCC-3'; RNU6-5'-TCGCTTCGGCAGCACATA T-3', universal reverse primers were provided in the cDNA synthesis kit; ACACB-F: CTGGAGAAGGGCGTCATATC, ACACB-R: CTGGATATGCACGTGACTCAG; FGFR1-F: TTAATACCACCGACAAAGAG, FGFR1-R: GTAGACGAT GACCGACCC; PPP3CA-F: TGGTCCCTTCCATTTGTT, PPP3CA-R: AAGCCTTTCAGCGTCAGC; SYK-F: GTT AGAGAAAGGAGAGCGGATG; SYK-R: TTCCTGTGA TTGCTCCTGTG; 18S-F: GTAACCCGTTGAACCCCATT; 18S-R: CCATCCAATCGGTAGTAGCG.

Identification of DEGs. Expression data were normalized by quantile normalization and the Robust Multichip Average (RMA) algorithm. The probe level was generated after normalization. The unpaired Student's t-test was used to identify genes that were differentially expressed between the miR-145-5p-overexpression group and the NC group. The fold-change (FC) value of gene expression between two groups was calculated, and genes with FC value $\geq 1.5$ and $\mathrm{P}$-value $<0.05$ were selected as DEGs.

Enrichment analysis of DEGs. The online tool DAVID (10) (version: 6.8, Database for Annotation, Visualization and Integrated Discovery) was used to perform the Gene Ontology (GO) functional and pathway enrichment analyses of DEGs based on GO database (11) and Kyoto Encyclopedia of Genes and Genomes (KEGG) PATHWAY database (12). The P-value of each term calculated by Fisher's exact test (13) was adjusted by the Benjamini-Hochberg method (14). Only function terms with adjusted $\mathrm{P}$-value $<0.05$ were considered significant.

Prediction of hsa-miR-145-5p target genes. Target genes of hsa-miR-145-5p were predicted from the DEGs identified above based on the miRNA-targeted interaction information in the miRWalk2.0 (15), miRDB (16), RNA22 (17), miRanda (18), RNAhybrid (19) and TargetScan (20) databases.

Functional analysis of hsa-miR-145-5p targets. The GO functions in the category of biological process (BP) and KEGG pathway enrichment analyses of the DEGs targeting by hsa-miR-145-5p were conducted using DAVID, and only function terms with adjusted P-value $<0.05$ were considered significant. Subsequently, the network consisting of GO and 
KEGG terms was visualized using the plugin ClueGO in Cytoscape (version 2.2.6) (21). Additionally, the network of significant pathways was visualized using the plugin CyKEGGParser (version 1.2.7) (22).

Construction of PPI network. The PPIs of the DEGs targeting by hsa-miR-145-5p were extracted from STRING database (version 10.0) (23) with default parameters. The PPIs meeting the combined score $>0.4$ were used to construct PPI network, which was then visualized by Cytoscape (version 3.4.0) (24). In PPI network, each node represents a protein and each edge between two nodes represents an interaction between these two proteins.

Furthermore, network topological features of nodes in the PPI network, including degree, betweenness and closeness, were analyzed by the plugin CytoNCA (version 2.1.6) (25). The hub nodes were revealed according to the scores of degree, betweenness and closeness.

\section{Results}

DEGs in hsa-miR-145-5p-overexpressed Tu-177 cells. To validate the miR-145-5p expression change between the miR-145-5p mimic and the NC transfected Tu-177 cells, the miR-145-5p expression level was determined by qPCR. The results showed that miR-145-5p was significantly upregulated in miR-145-5p mimic transfected cells than that in $\mathrm{NC}$ cells (Fig. 1A).

Based on the analysis of mRNA microarray data, a total of 1501 upregulated and 887 downregulated genes were identified in the hsa-miR-145-5p-overexpressed Tu-177 cells, compared with the NC cells. The heat map of these DEGs showed that the identified DEGs were able to distinguish the two group samples (Fig. 1B).

Potential functions of the DEGs. The upregulated DEGs were significantly enriched in 185 GO terms and 7 KEGG pathways, such as immune response, cell adhesion, and the pathway of neuroactive ligand-receptor interaction (Fig. 2A). Meanwhile, the downregulated DEGs were significantly enriched in 97 GO terms and 4 pathways, such as cell cycle, cellular protein catabolic process, and Wnt signaling pathway (Fig. 2B).

The predicted target genes of hsa-miR-145-5p. Among the identified DEGs, 164 upregulated and 221 downregulated genes were predicted to be targeted by hsa-miR-145-5p. These target genes were able to distinguish the hsa-miR-145-5p-overexpressedcells from the NC cells (Fig. 1C).

Furthermore, the potential functions of the DEGs targeting by hsa-miR-145-5p were analyzed to reveal the potential biological functions of hsa-miR-145-5p in LSCC. The upregulated target genes were significantly enriched in functions of immunity, such as the GO terms of negative regulation of lymphocyte proliferation, which interacted with the GO terms of regulation of interleukin-10 production. Furthermore, the pathway of cell adhesion molecules (CAMs) enriched by the upregulated target genes interacted with the pathway of leukocyte transendothelial migration, which was predicted to have a correlation with bicellular tight junction (Fig. 3A). Meanwhile, the downregulated target genes were significantly enriched in the p53 signaling

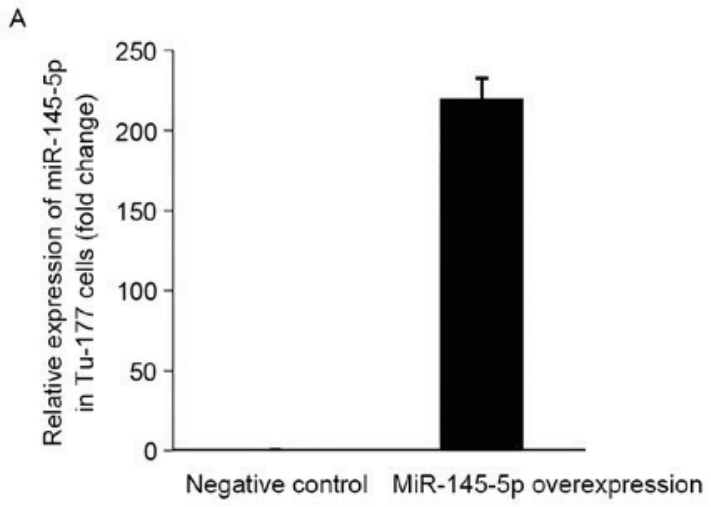

B

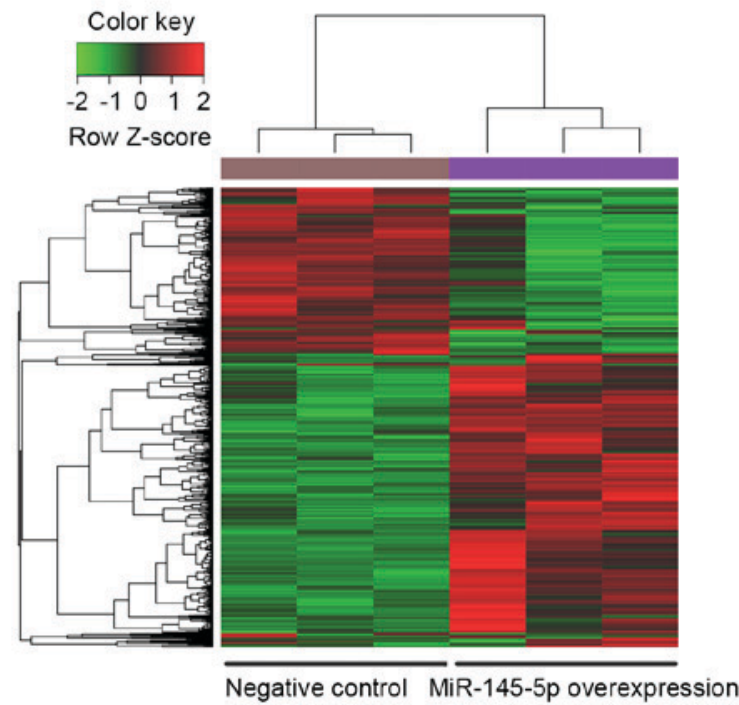

C

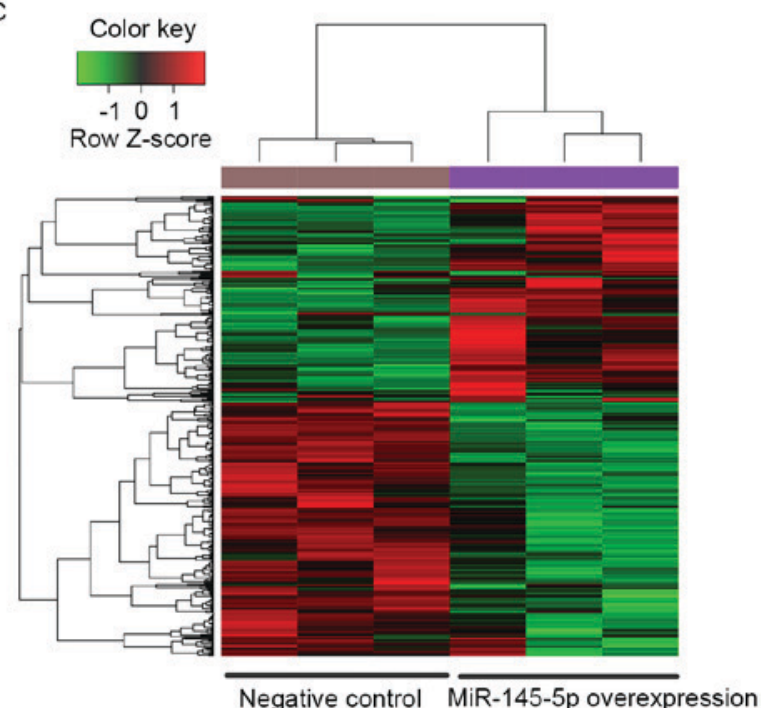

Figure 1. Microarray analysis of miR-145-5p-overexpressed human LSCC Tu-177 cells. (A) Expression level of miR-145-5p was determined by real-time quantitative polymerase chain reaction (RT-qPCR). Tu-177 cells were transfected with miR-145-5p mimic or negative control for $48 \mathrm{~h}$, respectively. Then total RNA was extracted and qPCR was performed. (B and C) Clustering analysis of differentially expressed genes. The heat maps showing the expression patterns of all differentially expressed genes (B) and differentially expressed target genes of miR-145-5p (C) in the miR-145-5p-overexpressed Tu-177 cells and the negative control cells Each column indicates a cell sample, and each row indicates a gene. Genes in red show upregulated expression, and genes in green show downregulated expression. Bars of RT-qPCR experiments represent the mean \pm standard deviation of three independent replicates. 

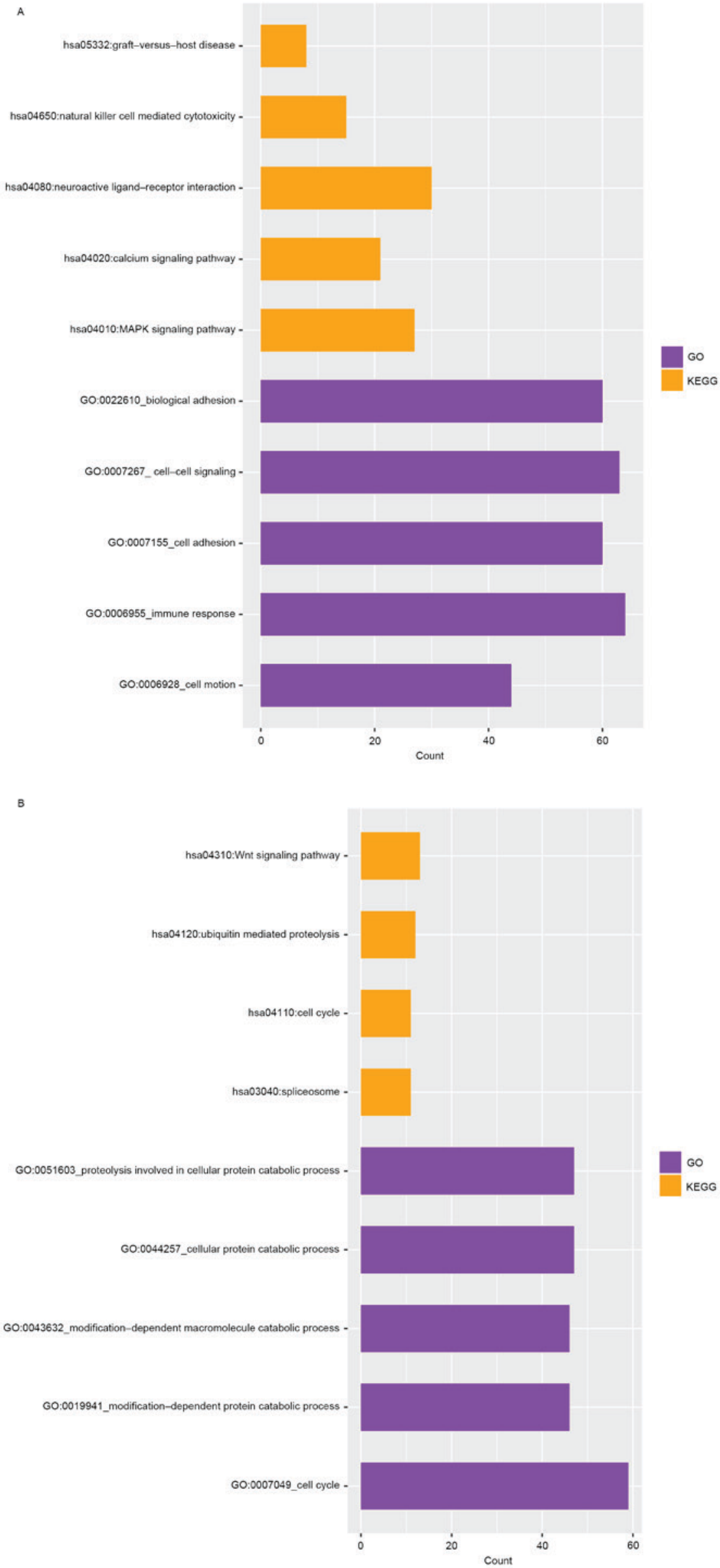

Figure 2. GO and KEGG pathway enrichment analysesof upregulated genes (A) and downregulated genes (B). GO, Gene Ontology; KEGG, Kyoto Encyclopedia of Genes and Genomes; MAPK, mitogen-activated protein kinase. 
pathway, and the GO term of cell-cell junction assembly which interacted with the GO term of cellular component disassembly involved in execution phase of apoptosis (Fig. 3B).

The PPI analysis of hsa-miR-145-5p target genes. To further investigate the potential PPIs of the DEGs targeted by hsa-miR-145-5p, a PPI network was constructed. This network consisted of 267 nodes and 416 PPI pairs. The upregulated $A C A C B$ had the highest degree and interacted with genes like the downregulated $P P P 3 C A$ and $S Y K$, as well as the upregulated FGFR1. PPP3CA interacted with 15 genes, such as MEF2A and FGFRI (Fig. 4).

The expression of ACACB, FGFR1, PPP3CA, and SYK in hsa-miR-145-5p-overexpressed Tu-177 cells. RT-PCR results revealed that the mRNA expressions of $A C A C B$ and FGFRI were obviously enhanced in miR-145-5p-overexpressed Tu-177 cells, while overexpressing miR-145-5p significantly reduced mRNA expression of PPP3CA and $S Y K$ (Fig. 5).

\section{Discussion}

In the present study, a total of 1,501 upregulated and 887 downregulated genes were identified in the hsa-miR-145-5p-overexpressed laryngeal squamous carcinoma Tu-177 cells, compared with the NC cells. Among these DEGs, 164 upregulated and 221 downregulated genes were predicted to be targeted by hsa-miR-145-5p. The upregulated target genes were mainly related to functions of immunity, whereas the downregulated target genes were significantly enriched in the p53 signaling pathway. In the PPI network, 267 target genes were included, and the upregulated $A C A C B$ had the highest degree. Besides, the mRNA expressions of $A C A C B$ and FGFRI were obviously enhanced in miR-145-5p-overexpressed Tu-177 cells, while overexpressing miR-145-5p significantly reduced mRNA expression of PPP3CA and $S Y K$.

$A C A C B$ encodes acetyl-CoA carboxylase (ACC) beta. ACC is a biotin-containing enzyme which catalyzes the carboxylation of acetyl-CoA to malonyl-CoA in fatty acid synthesis, and regulates fatty acid oxidation (26). In human colorectal cancer cells, fatty acid synthase suppression mediates the anticancer substance oridonin-induced apoptosis (27). Mak et al also found that ACC involved in lipid metabolism may be a potential anticancer target protein (28). Although there is no any evidence to prove the association of $A C A C B$ with LSCC or miR-145-5p, the results above indicate that miR-145-5p overexpression may induce $A C A C B$ expression in LSCC cells, which results in abnormity of fatty acid metabolism in LSCC.

In the PPI network, a set of DEGs were predicted to interact with $A C A C B$, such as the downregulated $P P P 3 C A$ and $S Y K$, as well as the upregulated FGFR1.PPP3CA encodes protein phosphatase 3 catalytic subunit alpha, also named calcineurin A alpha (29). Ostenfeld et al have reported that miR-145 induces cell death in human urothelial cancer cells by targeting PPP3CA, clathrin interactor 1 and core-binding factor $\beta$ subunit (30). Furthermore, PPP3CA has been also reported to be involved in the invasiveness of other cancers, such as breast cancer (31), pancreatic cancer (32), and small cell lung cancer (33). In the present study, PPP3CA was predicted to be correlated with the functions of cell cycle, which is commonly

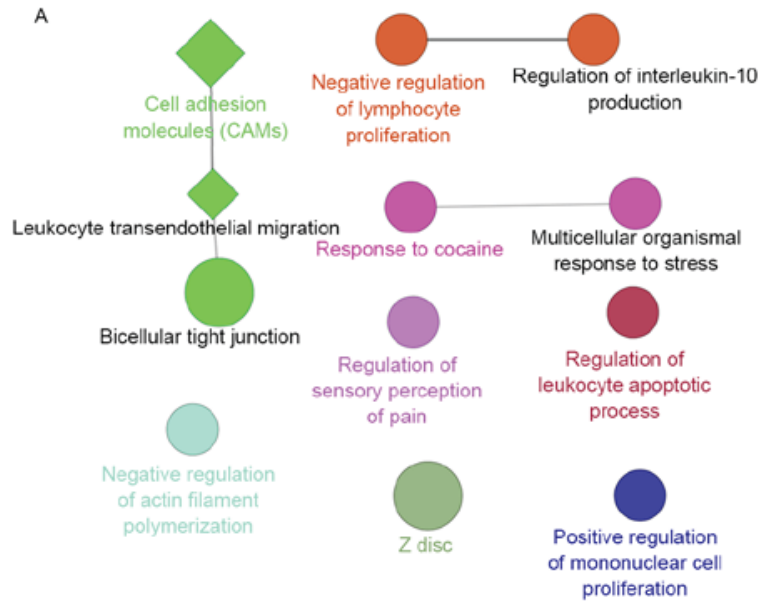

B

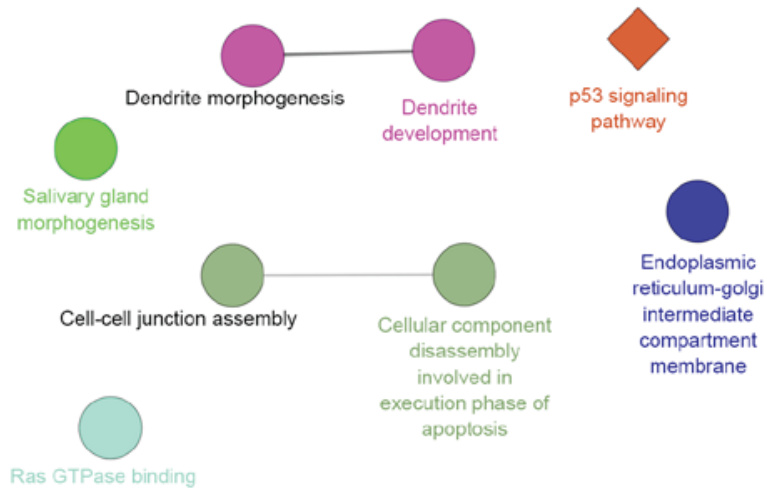

Figure 3. Interaction networks of GO and KEGG pathway terms enriched by upregulated genes (A) and downregulated genes (B). Round nodes represent GO terms, and diamonds represent KEGG pathway terms. GO, Gene Ontology; KEGG, Kyoto Encyclopedia of Genes and Genomes.

deregulated in cancers, including in LSCC. Collectively, miR-145-5p overexpression might induce the downregulation of PPP3CA, which likely affected the cell cycle and invasiveness of LSCC cells. $S Y K$ encodes spleen associated tyrosine kinase, which can couple activated immunoreceptors to downstream signaling pathways that regulate multiple cell progresses, such as cell proliferation, differentiation and phagocytosis (34). Currently, $S Y K$ has not been confirmed to be associated with LSCC or miR-145-5p. We speculated that miR-145-5p overexpression resulted in downregulation of $S Y K$, thus suppressing the cell proliferation of LSCC cells. FGFRI (fibroblast growth factor receptor 1) has been previously found to be frequently amplified in head and neck squamous cell carcinoma (HNSCC), and it has been identified as a candidate prognostic biomarker of HNSCC $(35,36)$. Furthermore, fibroblast growth factor receptor family member proteins (FGFR1-4) were recently reported to be also frequently highly expressed in oral cavity and oropharyngeal squamous cell carcinoma (37). In the PPI network, FGFRl interacted with $P P P 3 C A$, and the mRNA expressions of FGFRI and PPP3CA were obviously enhanced and reduced by overexpressing of miR-145-5p, respectively. Therefore, miR-145-5p overexpression may also change the expression of FGFR1 in LSCC cells via downregulating $P P P 3 C A$. 


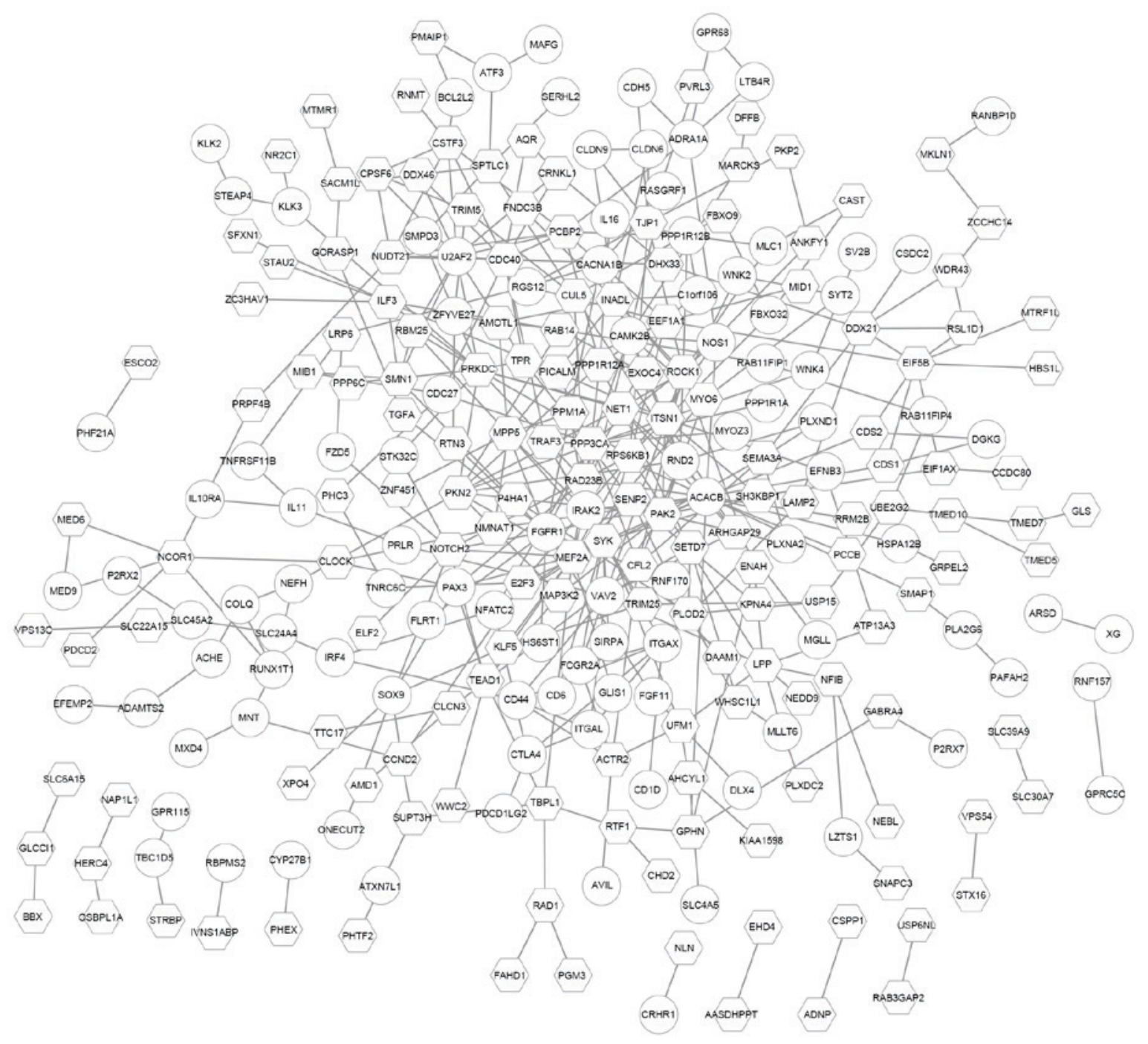

Figure 4. Protein-protein interaction network consisting of differentially expressed target genes of miR-145-5p. Round nodes represent upregulated genes, and quadrilateral nodes represent downregulated genes.

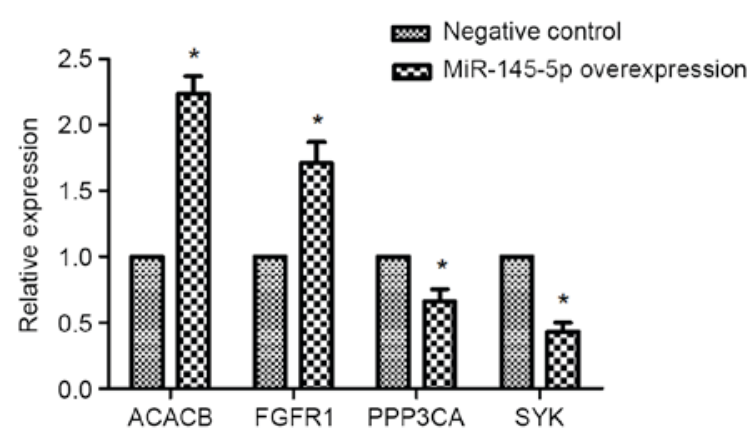

Figure 5. Expression levels of $A C A C B, F G F R 1, P P P 3 C A$ and $S Y K$ by RT-qPCR. Tu-177 cells were transfected with miR-145-5p mimic or negative control for $48 \mathrm{~h}$, respectively. " $\mathrm{P}<0.05$ vs. NC. RT-qPCR: real-time quantitative polymerase chain reaction.

Additionally, in this study, we also found that the upregulated target genes were mainly enriched in functions of immunity, such as regulation of lymphocyte proliferation and interleukin-10 production. The downregulated was significantly related to the p53 signaling pathway. In LSCC, lymphocytes are proliferated and lymphokines are overexpressed $(38,39)$.
Disruption of p53 function has been independently shown to occur in the majority of HNSCC (40). Recently, p53 has been discovered to mediate the amplification of the transcription of pro-apoptotic miRNAs in LSCC (41). Therefore, miR-145-5p overexpression may also changes immune responses and p53 signaling pathway to exert its anticancer efficiency in LSCC.

Despite the aforementioned results, this study still had several limitations. For example, the expression levels of the DEGs are needed to be confirmed, and the regulatory correlations between miR-145-5p and its predicted targets should also be validated by experiments. In our further study, we will confirm the predicted results in animal models and clinical samples.

In conclusion, based on microarray technology, we investigated expression changes of downstream genes of the miR-145-5p in human LSCC. A total of 1501 upregulated and 887 downregulated genes were identified in the hsa-miR-145-5p-overexpressed Tu-177 cells, compared with the NC cells. A series of genes were predicted to be targeted by hsa-miR-145-5p, and they were predicted to be related to immunity or p53 signaling pathway. Furthermore, some target 
genes were predicted to have interactions in the PPI network, such as $A C A C B$ that was associated with fatty acid metabolism, PPP3CA that was related to cell cycle and invasiveness, $S Y K$ that was correlated with cell proliferation. Therefore, hsa-miR-145-5p may function in LSCC via regulating multiple cell processes, such as cell proliferation and invasiveness, fatty acid metabolism, immunity and p53 signaling pathway. These findings provide novel information for the investigation of miR-145-5p functions and mechanisms in LSCC.

\section{Acknowledgements}

The present study was supported by the National Natural Science Foundation of China (grant nos. 81602394, 81572670, 81402256), Scientific and Technological Innovation Programs of Higher Education Institutions in Shanxi (STIP, 2016-92), the Research Project of Shanxi Province Health and Family Planning Commission (201301073, 2014028, 201601038, 201601037), the Excellent Talent Science and Technology Innovation Project of Shanxi Province (201605D211029), and China Postdoctoral Science Foundation (2016M591412), and the Shanxi Scholarship Council of China (2016-118).

\section{References}

1. Zhang SY, Lu ZM, Luo XN, Chen LS, Ge PJ, Song XH, Chen SH and $\mathrm{Wu}$ YL: Retrospective analysis of prognostic factors in 205 patients with laryngeal squamous cell carcinoma who underwent surgical treatment. PloS One 8: e60157, 2013.

2. Siegel RL, Miller KD and Jemal A: Cancer statistics, 2016. CA Cancer J Clin 66: 7-30, 2016.

3. Slattery ML, Herrick JS, Mullany LE, Valeri N, Stevens J, Caan BJ, Samowitz W and Wolff RK: An evaluation and replication of miRNAs with disease stage and colorectal cancer-specific mortality. Int J Cancer 137: 428-438, 2015.

4. Letelier P, García P, Leal P, Álvarez H, Ili C, López J, Castillo J, Brebi P and Roa JC: miR-1 and miR-145 act as tumor suppressor microRNAs in gallbladder cancer. Int J Clin Exp Pathol 7: $1849-1867,2014$

5. Andersen M, Grauslund M, Ravn J, Sørensen JB, Andersen CB and Santoni-Rugiu E: Diagnostic potential of miR-126, miR-143, miR-145, and miR-652 in malignant pleural mesothelioma. J Mol Diagn 16: 418-430, 2014

6. Ozen M, Karatas OF, Gulluoglu S, Bayrak OF, Sevli S, Guzel E, Ekici ID, Caskurlu T, Solak M, Creighton CJ and Ittmann M: Overexpression of miR-145-5p inhibits proliferation of prostate cancer cells and reduces SOX2 expression. Cancer Invest 33: 251-258, 2015

7. Karatas OF, Yuceturk B, Suer I, Yilmaz M, Cansiz H, Solak M, Ittmann M and Ozen M: Role of miR-145 in human laryngeal squamous cell carcinoma. Head Neck 38: 260-266, 2016.

8. Karatas OF, Suer I, Yuceturk B, Yilmaz M, Hajiyev Y, Creighton CJ, Ittmann $\mathrm{M}$ and Ozen M: The role of miR-145 in stem cell characteristics of human laryngeal squamous cell carcinoma Hep-2 cells. Tumor Biol 37: 4183-4192, 2016.

9. WeiGao CZ, Chen G, Li Y, Wen S, Huang F and Wang B: Novel mechanism and clinical significance of Hsa-miR-145-5P/FSCN1 regulatory axis in laryngeal squamous cell carcinoma. In: Tumor academic conference across the Taiwan Straits, 2014.

10. Huang DW, Sherman BT and Lempicki RA: Systematic and integrative analysis of large gene lists using DAVID bioinformatics resources. Nat Protoc 4: 44-57, 2008.

11. Gene Ontology Consortium: Gene ontology consortium: Going forward. Nucleic Acids Res 43 (Database issue): D1049-D1056, 2015.

12. Kanehisa M and Goto S: KEGG: Kyoto encyclopedia of genes and genomes. Nucleic Acids Res 28: 27-30, 2000.

13. Preacher KJ and Briggs NE: Calculation for Fisher's Exact Test. Retrieved, 2015.

14. Benjamini Y and Hochberg Y: Controlling the false discovery rate: A practical and powerful approach to multiple testing. J Royal Stat Soci Series B (Methodological) 57: 289-300, 1995.
15. Dweep H, Sticht C, Pandey P and Gretz N: miRWalk-database: Prediction of possible miRNA binding sites by 'walking' the genes of three genomes. J Biomed Inform 44: 839-847, 2011.

16. Wong $N$ and Wang X: miRDB: An online resource for microRNA target prediction and functional annotations. Nucleic Acids Res 43 (Database issue): D146-D152, 2015.

17. Miranda KC, Huynh T, Tay Y, Ang YS, Tam WL, Thomson AM, Lim B and Rigoutsos I: A pattern-based method for the identification of microRNA binding sites and their corresponding heteroduplexes. Cell 126: 1203-1217, 2006.

18. Betel D, Wilson M, Gabow A, Marks DS and Sander C: The microRNA.org resource: Targets and expression. Nucleic Acids Res 36 (Database issue): D149-D153, 2008.

19. Kruger J and Rehmsmeier M: RNAhybrid: microRNA target prediction easy, fast and flexible. Nucleic Acids Res 34 (Web Server issue): W451-W454, 2006.

20. Lewis BP, Shih IH, Jones-Rhoades MW, Bartel DP and Burge CB: Prediction of mammalian microRNA targets. Cell 115: 787-798, 2003.

21. Bindea G, Mlecnik B, Hackl H, Charoentong P, Tosolini M, Kirilovsky A, Fridman WH, Pagès F, Trajanoski $\mathrm{Z}$ and Galon J: ClueGO: A Cytoscape plug-in to decipher functionally grouped gene ontology and pathway annotation networks. Bioinformatics 25: 1091-1093, 2009.

22. Nersisyan L, Samsonyan R and Arakelyan A: CyKEGGParser: Tailoring KEGG pathways to fit into systems biology analysis workflows. F1000Res 3: 145, 2014

23. Szklarczyk D, Franceschini A, Wyder S, Forslund K, Heller D, Huerta-Cepas J, Simonovic M, Roth A, Santos A, Tsafou KP, et al: STRING v10: Protein-protein interaction networks, integrated over the tree of life. Nucleic Acids Res 43 (Database issue): D447-D452, 2015.

24. Shannon P, Markiel A, Ozier O, Baliga NS, Wang JT, Ramage D, Amin N, Schwikowski B and Ideker T: Cytoscape: A software environment for integrated models of biomolecular interaction networks. Genome Res 13: 2498-2504, 2003.

25. Tang Y, Li M, Wang J, Pan Y and Wu FX: CytoNCA: A cytoscape plugin for centrality analysis and evaluation of protein interaction networks. Biosystems 127: 67-72, 2015.

26. Saddik M, Gamble J, Witters LA and Lopaschuk GD: Acetyl-CoA carboxylase regulation of fatty acid oxidation in the heart. $\mathrm{J}$ Biol Chem 268: 25836-25845, 1993.

27. Kwan HY, Yang Z, Fong WF, Hu YM, Yu ZL and Hsiao WL: The anticancer effect of oridonin is mediated by fatty acid synthase suppression in human colorectal cancer cells. Journal of Gastroenterology 48: 182-192, 2013.

28. Mak L, Liggi S, Tan L, Kusonmano K, Rollinger JM, Koutsoukas A, Glen RC and Kirchmair J: Anti-cancer drug development: Computational strategies to identify and target proteins involved in cancer metabolism. Curr Pharm Des 19: 532-577, 2013.

29. Wang MG, Yi H, Guerini D, Klee CB and Mcbride OW: Calcineurin A alpha (PPP3CA), calcineurin A beta (PPP3CB) and calcineurin B (PPP3R1) are located on human chromosomes 4, 10q21 $\square$ q22 and 2 p16 $\square$ p15 respectively. Cytogenet Cell Genet 72: 236-241, 1996.

30. Ostenfeld MS, Bramsen JB, Lamy P, Villadsen SB, Fristrup N, Sørensen KD, Ulhøi B, Borre M, Kjems J, Dyrskjøt L and Orntoft TF: miR-145 induces caspase-dependent and -independent cell death in urothelial cancer cell lines with targeting of an expression signature present in Ta bladder tumors. Oncogene 29: 1073-1084, 2010 .

31. Gabrovska PN, Smith RA, Haupt LM and Griffiths LR: Investigation of two Wnt signalling pathway single nucleotide polymorphisms in a breast cancer-affected Australian population. Twin Res Hum Genet 14: 562-567, 2011.

32. Buchholz M, Schatz A, Wagner M, Michl P, Linhart T, Adler G, Gress TM and Ellenrieder V: Overexpression of c-myc in pancreatic cancer caused by ectopic activation of NFATc1 and the Ca2+/calcineurin signaling pathway. Embo J 25: 3714-3724, 2006.

33. Liu Y, Zhang Y, Min J, Liu LL, Ma NQ, Feng YM, Liu D, Wang PZ, Huang DD, Zhuang Y and Zhang HL: Calcineurin promotes proliferation, migration, and invasion of small cell lung cancer. Tumor Biol 31: 199-207, 2010.

34. Buchner M, Fuchs S, Prinz G, Pfeifer D, Bartholomé K, Burger M, Chevalier N, Vallat L, Timmer J, Gribben JG, et al: Spleen tyrosine kinase is overexpressed and represents a potential therapeutic target in chronic lymphocytic leukemia. Cancer Res 69: 5424-5432, 2009 . 
35. Göke F, Bode M, Franzen A, Kirsten R, Goltz D, Göke A Sharma R, Boehm D, Vogel W, Wagner P, et al: Fibroblast growth factor receptor 1 amplification is a common event in squamous cell carcinoma of the head and neck. Mod Pathol 26: 1298-1306, 2013.

36. Koole K, Brunen D, van Kempen PM, Noorlag R, de Bree R, Lieftink C, van Es RJ, Bernards R and Willems SM: FGFR1 Is a potential prognostic biomarker and therapeutic target in head and neck squamous cell carcinoma. Clin Cancer Res 22: 3884-3893, 2016.

37. Koole K, Clausen MJ, van Es RJ, van Kempen PM, Melchers LJ, Koole R, Langendijk JA, van Diest PJ, Roodenburg JL, Schuuring E and Willems SM: FGFR family members protein expression as prognostic markers in oral cavity and oropharyngeal squamous cell carcinoma. Mol Diagn Ther 20: 363-374, 2016.

38. Bergmann C, Strauss L, Zeidler R, Lang S and Whiteside TL: Expansion of human $\mathrm{T}$ regulatory type 1 cells in the microenvironment of cyclooxygenase 2 overexpressing head and neck squamous cell carcinoma. Cancer Res 67: 8865-8873, 2007.
39. Jebreel A, Mistry D, Loke D, Dunn G, Hough V, Oliver K, Stafford N and Greenman J: Investigation of interleukin 10, 12 and 18 levels in patients with head and neck cancer. J Laryngol Otol 121: 246-252, 2007.

40. Allen CT, Ricker JL, Chen Z and Van Waes C: Role of activated nuclear factor-kappaB in the pathogenesis and therapy of squamous cell carcinoma of the head and neck. Head Neck 29: 959-971, 2007.

41. Kan X, Sun Y,Lu J, Li M, Wang Y, Li Q, Liu Y, Liu M and Tian L: Co-inhibition of miRNA-21 and miRNA-221 induces apoptosis by enhancing the p53-mediated expression of pro-apoptotic miRNAs in laryngeal squamous cell carcinoma. Mol Med Rep 13: 4315-4320, 2016 